\title{
Differences in Correlation between Subjective and Measured Olfactory and Gustatory Dysfunctions after Initial Ear, Nose and Throat Evaluation
}

\author{
Hans Jacob Nørgaard ${ }^{1,2}$ Alexander Wieck Fjaeldstad ${ }^{1,2,3}$ (우 \\ ${ }^{1}$ Department of Otorhinolaryngology, Flavour Clinic, West Hospital \\ Unit, Central Denmark Region, Holstebro, Denmark \\ 2 Flavour Institute, Aarhus University, Palle Juul-Jensens, Aarhus N, \\ Denmark \\ ${ }^{3}$ Department of Psychiatry, Center for Eudaimonia and Human \\ Flourishing, University of Oxford, Oxford, United Kingdom
}

\author{
Address for correspondence Alexander Wieck Fjaeldstad, MD, PhD, \\ Department of Otorhinolaryngology, West Hospital Unit, Central \\ Denmark Region, Laegaardsvej 12, 7500 Holstebro, Denmark \\ (e-mail: Alefja@rm.dk).
}

Int Arch Otorhinolaryngol 2021;25(4):e563-e569.

\begin{abstract}
Keywords

- smell

- taste

- olfactory testing

- gustatory testing

- chemosensory dysfunction

Introduction Subjective chemosensory function can differ from measured function. Previous studies on olfactory assessment have found a positive correlation between subjective and measured scores. However, information on gustatory correlation between measured and subjective functions is sparse in patients who have undergone an initial ear, nose and throat (ENT) evaluation.

Objectives To evaluate the correlation between subjective and measured olfactory and gustatory dysfunctions in a population complaining of taste and/or smell dysfunction after an initial ENT evaluation without chemosensory testing. Furthermore, we aimed to assess the need for chemosensory testing depending on the type of subjective chemosensory dysfunction.

Methods A case series in which subjective chemosensory function was assessed through a questionnaire and measured chemosensory function was assessed by validated clinical tests.

Results In total, 602 patients with complaints of olfactory and/or gustatory dysfunction were included. We found that $50 \%$ of the patients with normal gustatory function and an olfactory impairment classified their olfactory impairment as a subjective taste disorder. Furthermore, $98 \%$ of the patients who rated their olfactory function as absent did have a measurable olfactory impairment, but only $64 \%$ were anosmic.

Conclusion Subjective gustatory dysfunction was poorly correlated with measured gustatory dysfunction, and was often found to reflect olfactory dysfunction. Contrarily, subjective olfactory dysfunction was positively correlated with measurable olfactory dysfunction. Although subjective anosmia was a strong indicator of measured anosmia or hyposmia, the existence of remaining olfactory function was frequently found in these patients. Validated chemosensory testing should be performed in patients with perceived olfactory or gustatory deficits, as this could help ensure increased diagnostic precision and a relevant treatment.
\end{abstract}

received

September 2, 2020

accepted

November 8, 2020

published online

February 19, 2021
DOI https://doi.org/

10.1055/s-0040-1722249.

ISSN 1809-9777.

\footnotetext{
(C) 2021. Fundação Otorrinolaringologia. All rights reserved.

This is an open access article published by Thieme under the terms of the Creative Commons Attribution-NonDerivative-NonCommercial-License, permitting copying and reproduction so long as the original work is given appropriate credit. Contents may not be used for commercial purposes, or adapted, remixed, transformed or built upon. (https://creativecommons.org/ licenses/by-nc-nd/4.0/)

Thieme Revinter Publicações Ltda., Rua do Matoso 170, Rio de Janeiro, RJ, CEP 20270-135, Brazil
} 


\section{Introduction}

Although the sensory input from eating is often described as 'taste', the perception of flavour is truly a multisensory experience. Thus, if any sensory component is reduced or lost, it can have sweeping effects on perception. Olfactory dysfunction is the most common sensory loss, but its symptoms are often mistakenly ascribed to an impairment in the gustatory function, since the two senses are interrelated. ${ }^{1}$ This is in part due to the fact that 'taste of substances' is mainly driven by aromas, which are perceived by the olfactory system.

Gustatory function consists of at least five basic taste qualities; sweet, salty, bitter, sour, and umami. ${ }^{2}$ The simultaneous olfactory stimulation of retronasal aromas are often not perceived as a contribution from the nose, which makes it difficult to distinguish between these senses. ${ }^{3}$

Olfactory disorders are common, affecting up to $20 \%$ of the population. ${ }^{4}$ They can lead to decreased quality of life, ${ }^{5}$ depression, ${ }^{6}$ increased health risk (ingestion of rotten food, and lack of response to fires and gas leaks, for example), and malnutrition. ${ }^{7}$ Self-rating of the olfactory function is often used in the clinical practice. ${ }^{8}$ Specifically, subjective reduction or loss of smell constitutes one of the four diagnostic criteria for chronic rhinosinusitis. ${ }^{9}$ Although the updated guidelines on chronic rhinosinusitis advise the performance of olfactory testing, ${ }^{10}$ this is not always done routinely for both taste and smell functions. Correct assessment of the chemosensory function is an important factor in diagnosis and treatment; thus it is crucial to examine the quality of the self-assessment.

Diagnostic tests can be applied to determine the olfactory and gustatory functions. Yet, thorough testing is time-consuming. Therefore, it is of great importance to consider whether or not self-assessment is a valid predictor of olfactory and gustatory dysfunction compared with diagnostic testing. Previous studies ${ }^{11,12}$ have established a positive correlation between self-assessment of olfactory abilities and olfactory function, while others ${ }^{13}$ demonstrated a poor correlation between these parameters. Information on the gustatory correlation between the measured and subjective functions is sparse. Due to the confusion between taste and smell, an evaluation of both senses is a prerequisite to untangle the assessment of these ambiguous sensory assessments.

The aim of the present study was to evaluate the correlation between subjective and measured olfactory and gustatory dysfunctions in referred patients complaining of taste and/or smell dysfunction to identify the need of chemosensory testing. Moreover, the aim was also to assess the need for chemosensory testing depending on the subjective evaluation.

\section{Materials and Methods}

\section{Patients}

Patients with complaints of olfactory and or gustatory dysfunction were included in a Research Electronic Data Capture (REDCap, Vanderbilt University, Nashville, Tennessee, USA) registry from January 2017 to April 2019. Patients were referred from either a private ear, nose and throat (ENT) specialist or an ENT department to the Flavour Clinic (a Danish specialized national out-patient clinic for taste and smell disorder) for diagnostics and treatment. All referred patients were eligible for inclusion in the registry, and no patients declined inclusion. A previous ${ }^{13}$ study has been published in which the diagnostic workflow is more thoroughly described. A part of the data of the current study was also included in this previous study; however, not the details of the association between the subjective and measured functions, which is the primary focus of the current study.

Prior to referral, the patients had been examined by an ENT specialist and undergone inspection of the nasal cavity and rhino pharynx, allergy testing, and computed tomography of the nasal cavity and paranasal sinuses.

\section{Testing Procedure}

Prior to testing, patients filled out a questionnaire on demographics and self-rated olfactory and gustatory functions (good, normal, reduced, or absent). The patients avoided smoking, eating, and drinking (with the exception of water) one hour before testing. The Mini-Mental State Examination (MMSE) was applied to all patients to ensure sufficient cognitive capacity during the testing, and the 22-item Sinonasal Outcome Test (SNOT22) was applied to quantify sinonasal symptoms.

\section{Olfactory Testing}

The olfactory function was examined with the full version of the Sniffin' Sticks olfactory test. ${ }^{14}$ This test includes the assessment of the olfactory threshold $(\mathrm{T})$, discrimination (D), and identification (I) score, which generates a total TDI score (1-48 points). A Danish version of this test has been validated, ${ }^{15}$ making it applicable for research and clinical work in Denmark.

The total individual TDI score was computed, and the participants were separated into 3 groups; normosmia (TDI $\geq 30$ ), hyposmia (TDI bertween 15 and 29), and anosmia $(\mathrm{TDI} \leq 16) .{ }^{16}$ The cut-off value for hyposmia is based on normative data on healthy individuals with subjective normal olfactory function from a Danish population, in accordance to the test guidelines. ${ }^{15}$

\section{Gustatory Testing}

The clinical pipeline for gustatory testing was changed during the course of the present study. As such, gustatory testing was conducted in two different ways. Initially, the Taste Strips examination was applied. ${ }^{17}$ As some patients had difficulties with this test, a study was conducted to assess the re-test reliability of the Taste Strips and another gustatory test, the Taste-Drop-Test. As the Taste-Drop-Test proved more reliable, ${ }^{18}$ it was subsequently used.

\section{Test Procedures}

Taste Strips Test

Taste strips are filter paper strips impregnated with taste solutions representative of four basic tastants at four 
different concentrations (sweet: $0.4 \mathrm{~g} / \mathrm{mL}, 0.2 \mathrm{~g} / \mathrm{mL}, 0.1 \mathrm{~g} / \mathrm{mL}$, and $0.05 \mathrm{~g} / \mathrm{mL}$ of sucrose; sour: $0.3 \mathrm{~g} / \mathrm{mL}, 0.165 \mathrm{~g} / \mathrm{mL}, 0.09$ $\mathrm{g} / \mathrm{mL}$, and $0.05 \mathrm{~g} / \mathrm{mL}$ of citric acid; salty: $0.25 \mathrm{~g} / \mathrm{mL}, 0.1 \mathrm{~g} / \mathrm{mL}$, $0.04 \mathrm{~g} / \mathrm{mL}$, and $0.016 \mathrm{~g} / \mathrm{mL}$ of sodium chloride; bitter: 0.006 $\mathrm{g} / \mathrm{mL}, 0.0024 \mathrm{~g} / \mathrm{mL}, 0.0009 \mathrm{~g} / \mathrm{mL}$, and $0.0004 \mathrm{~g} / \mathrm{mL}$ of quininehydrochloride). The Taste Strips test validated to assess gustatory sensitivity. It is administered in a pseudo-randomized manner in increasing concentrations, resulting in a total taste score ranging from 0 to 16 points for each patient. For a more detailed description of the Taste Strips, see Mueller et al. ${ }^{17}$

\section{The Taste-Drop-Test}

The Taste-Drop-Test consists of five different options of tastants (salty, sour, bitter, neutral, and sweet). One drop of the given tastant was applied on each side of the tongue with a transfer pipette. Subsequently, the patients had to select which tastant was applied before rinsing the mouth with water and repeating the procedure. The tastants were semi-randomized and presented in increasing concentrations until the correct identification, after which a staircase confirmation of sensitivity level for each tastant was applied. The total taste score (from 0 to 40 points) was established for each tested patient. For a more detailed description of the Taste-Drop-Test, see and Fjaeldstad et. al. ${ }^{18}$

\section{Cut-off values for Gustatory Testing}

For the Taste-Drop-Test and Taste Strips test, total taste scores below 25 and 9 points defined hypogeusia respectively. This cut-off value was defined as the 10th percentile of normative values. Ageusia, for the Taste-Drop-Test and Taste Strips, was defined as scores below 18 and 4 respectively. ${ }^{18}$

\section{Statistics}

Statistical analyses were completed using JMP (SAS Institute, Cary, NC, US) software, version 14.0. The mean total TDI scores for subjective olfactory ratings were compared using a twotailed $t$-test. The Spearman rank correlation coefficient $(\rho)$ was used to investigate possible correlations. The ability to classify loss of taste or smell was assessed by calculating the area under the curve (AUC) in receiver operating characteristic (ROC) curves. This was calculated for subjective ratings and sensory loss diagnosed with olfactory or gustatory testing.

The $\alpha$ level of statistical significance was set at 0.05 .

\section{Results}

A total of 602 patients were tested with the Sniffin' Sticks olfactory test. A random sample of these patients $(n=258)$ also underwent gustatory testing. In total, $55 \%(n=143)$ and $45 \%(n=115)$ of patients were tested with the Taste Strips and Taste-Drop-Test, respectively.

The measured olfactory impairments were frequent, with a mean total TDI score of 18.0 (95\% confidence interval [95\% $\mathrm{CI}$ ]: 17.4 to 18.7). A high proportion of patients was found to be anosmic $(45.3 \%, n=273)$ or hyposmic $(44.4 \%, n=267)$. Subjective olfactory impairment was also frequent, as patients often rated themselves as anosmic (57.3\%, $n=345)$ or hyposmic $(39.5 \%, n=238)$.
The measured gustatory impairments occurred less frequently, as $24.8 \%$ ( $n=64)$ of patients were hypogeusic, while only $10.5 \%(n=27)$ were ageusic. However, subjective ratings of hypogeusia $(55.8 \%, n=144)$ and ageusia $(29.8 \%, n=77$ ) were more frequent than the measured reduced gustatory function, as shown in - Table 1.

The mean MMSE score of the patients was of 28.4 (95\%CI: 28.3 to 28.6). Only 4 patients scored below 24 points, indicating a cognitive deficit.

Patients had a mean SNOT-22 score of 21.8 (95\%CI: 20.6 to 23.0), which was not statistically correlated to the olfactory TDI score $(\rho=0.0251 ; p=0.5401)$.

\section{Association between Olfactory and Gustatory Self- ratings and Measured Test Results}

Patients with a absent or reduced subjective sense of taste (85.7\%, $n=221)$ often had a normal taste test score $(n=138$; $62.4 \%)$. Most patients with subjective loss of taste and normal taste tests had a measurable olfactory deficit $(n=121 ; 87.7 \%)$. Contrarily, among the patients with a reduced or absent selfrated olfactory function and a normal olfactory test, only $2.7 \%$ $(n=7)$ were identified as hypogeusic or ageusic when tested.

In total, $81.0 \%(n=209)$ of the patients who had undergone olfactory and gustatory testing had a combined subjective smell and taste deficit (either reduced or absent), as shown in -Fig. 1a. However, only $28.3 \%(n=73)$ had a combined measurable loss of taste and smell, as shown in - Table 1. Of the 258 (100\%) patients who had undergone taste and smell tests, only 18 (7.0\%) had an isolated loss of taste, while 149 (57.8\%) had an isolated loss of smell, as shown in - Fig. 1 b.

\section{Accuracy of the Subjective Olfactory Loss}

The subjective assessment of the olfactory function was a moderate measurement for olfactory function based on the olfactory Sniffin' Sticks score (ROC AUC: 0.7951), as shown in - Fig. 2a. Patients with subjective anosmia had a statistically significant lower average mean TDI score compared with the subjective hyposmic group (mean difference: 7.5; $p<0.001$; two-tailed $t$-test).

In total, $98.3 \%$ of the patients with an olfactory selfassessment rated as absent did have a measurable reduction in olfactory function.

Only 19 patients were subjectively normosmic, which, for $84.2 \%$ of patients corresponded with a normal TDI score. However, out of the 345 (57.3\%) patients who rated their olfactory function as absent, 123 (35.6\%) scored within the normosmic or hyposmic range. As such, a third of the patients with a subjective belief of an inability to perceive odour were in fact able to detect smell.

Accuracy of Subjective Gustatory Function in Patients In total, $54.5 \%(n=42)$ of the patients with subjective ageusia and $66.7 \%(n=96)$ of the patients with subjective hypogeusia scored within the normogeusic range. The subjective assessment of gustatory function was a poor measurement for gustatory function based on the Taste-Drop-Test (ROC AUC: 0.5802) and Taste Strips test (ROC AUC: 0.5436 ), as shown in - Fig. $\mathbf{2 b , c}$. 
Table 1 Demographics of the study sample

\begin{tabular}{|c|c|c|c|c|}
\hline \multicolumn{5}{|c|}{ Olfaction and subjective olfactory function } \\
\hline Subjective olfactory function & All $(n=602)$ & Absent $(n=345)$ & Reduced $(n=238)$ & Normal/Good $(n=19)$ \\
\hline Age, years $(I Q R)^{\dagger}$ & $59(49-68)$ & $61(49-70)$ & $58(50-66)$ & $53(42-61)$ \\
\hline Gender: male, $n$ (\%) & $239(40)$ & $130(38)$ & $103(43)$ & $6(32)$ \\
\hline Total TDI score, mean (SD) & $18.0(7.9)$ & $14.5(6.1)$ & $22.0(7.4)$ & $32.2(2.6)$ \\
\hline Normosmic score, $n(\%)$ & $62(10.3)$ & $6(1.7)$ & $40(16.8)$ & $16(84.2)$ \\
\hline Hyposmic score, $n$ (\%) & $267(44.4)$ & $117(33.9)$ & $147(61.7)$ & $3(15.8)$ \\
\hline Anosmic score, $n(\%)$ & $273(45.3)$ & $222(64.4)$ & $51(21.4)$ & $0(0.0)$ \\
\hline Taste Strips test, mean (SD); $n=143$ & $11.8(4.1)$ & $12.5(3.9)$ & $11.3(4.0)$ & $6.0(2.5)$ \\
\hline Taste-Drop-Test, mean (SD); $n=115$ & $23.4(6.6)$ & $23.2(6.9)$ & $24.2(6.2)$ & $21.4(5.8)$ \\
\hline Normogeusic score, $n(\%)$ & $167(64.7)$ & $105(69.1)$ & $60(65.9)$ & $2(13.3)$ \\
\hline Hypogeusic score ,n (\%) & $64(24.8)$ & $28(18.4)$ & $25(27.5)$ & $11(73.4)$ \\
\hline Ageusic score, $n(\%)$ & $27(10.5)$ & $19(12.5)$ & $6(6.6)$ & $2(13.3)$ \\
\hline \multicolumn{5}{|c|}{ Gustation and subjective gustatory function } \\
\hline Subjective gustatory function & All $(n=258)$ & Absent $(n=77)$ & Reduced $(n=144)$ & Normal/Good $(n=37)$ \\
\hline Age, years $(\mathrm{IQR})^{\dagger}$ & $60(49-70)$ & $65(55-74)$ & $58(48-68)$ & $58(38-65)$ \\
\hline Gender: male/female, $n$ (\%) & $99(38)$ & $25(32)$ & $57(40)$ & $17(46)$ \\
\hline Taste Strips test, mean (SD) & $11.8(4.9)$ & $11.1(4.8)$ & $11.7(4.0)$ & $13.7(2.1)$ \\
\hline Taste-Drop-Test, mean (SD) & $23.4(6.6)$ & $22.0(7.1)$ & $24.2(6.4)$ & $23.9(6.3)$ \\
\hline Normogeusic score, $n(\%)$ & $167(64.7)$ & $42(54.5)$ & $96(66.7)$ & $29(78.4)$ \\
\hline Hypogeusic score, $n$ (\%) & $64(24.8)$ & $20(26.0)$ & $38(26.4)$ & $6(16.2)$ \\
\hline Ageusic score, $n$ (\%) & $27(10.5)$ & $15(19.5)$ & $10(6.9)$ & $2(5.4)$ \\
\hline Total TDI score, mean (SD) & $18.6(8.4)$ & $18.1(7.5)$ & $19.3(9.1)$ & $17.1(7.3)$ \\
\hline Normosmic score, $n$ (\%) & $36(14.0)$ & $8(10.4)$ & $27(18.8)$ & $1(2.7)$ \\
\hline Hyposmic score, $n(\%)$ & $108(41.9)$ & $36(46.7)$ & 56 (38.9) & $16(43.3)$ \\
\hline Anosmic score, $n(\%)$ & $114(44.2)$ & 33 (42.9) & $61(42.4)$ & $20(54.0)$ \\
\hline
\end{tabular}

Abbreviations: IQR, interquartile range; SD, standard deviation; TDI, threshold, discrimination and identification.

Notes: Normosmia, hyposmia and anosmia were computed from the total TDI score. Normogeusic, hypogeusic, and ageusic scores were computed from the total score of the Taste Strips test or Taste-Drop-Test.

${ }^{\dagger}$ Median age.

\section{Discussion}

\section{Key Findings}

We found that the subjective olfactory function was a moderate predictor of the measured olfactory functionthat the , and subjective gustatory function did not reflect the measured gustatory abilities. Furthermore, the subjective gustatory dysfunction was associatied with the measured olfactory dysfunction.

Moreover, patients complaining of complete anosmia often had a measurable olfactory impairment.

\section{Comparisons with Other Studies}

Accuracy of Subjective Olfaction in Patients

Patients with an absent subjective sense of smell had a statistically significant lower average TDI score compared with patients with a reduced subjective sense of smell. The subjective assessment of olfactory function was associated with the Sniffin' Sticks score (ROC AUC: 0.7951). In agreement with those findings, ${ }^{11,12}$ other studies a reported similar moderate correlation $(\rho=0.55$ to 0.57$)$ between self-assessment and measured olfaction in patients. These findings suggest that patients with a subjective loss of smell are, to some extent, aware of the degree of olfactory impairment.

We also found that $98.3 \%(n=339)$ of patients who rated their olfactory function as absent scored within the anosmic or hyposmic range. This result clearly indicates that patients categorizing themselves as having an absent sense of smell often appear to suffer from some degree of olfactory impairment. Contrarily, ${ }^{20}$ a recent study found that $28.9 \%$ of their anosmic patient group rated their olfactory function as at least average. This suggests that a noticeable share of anosmic patients are in fact unaware of such a deficit. These findings are in line with those of Shu et al., ${ }^{21}$ who found that $36 \%$ of their 

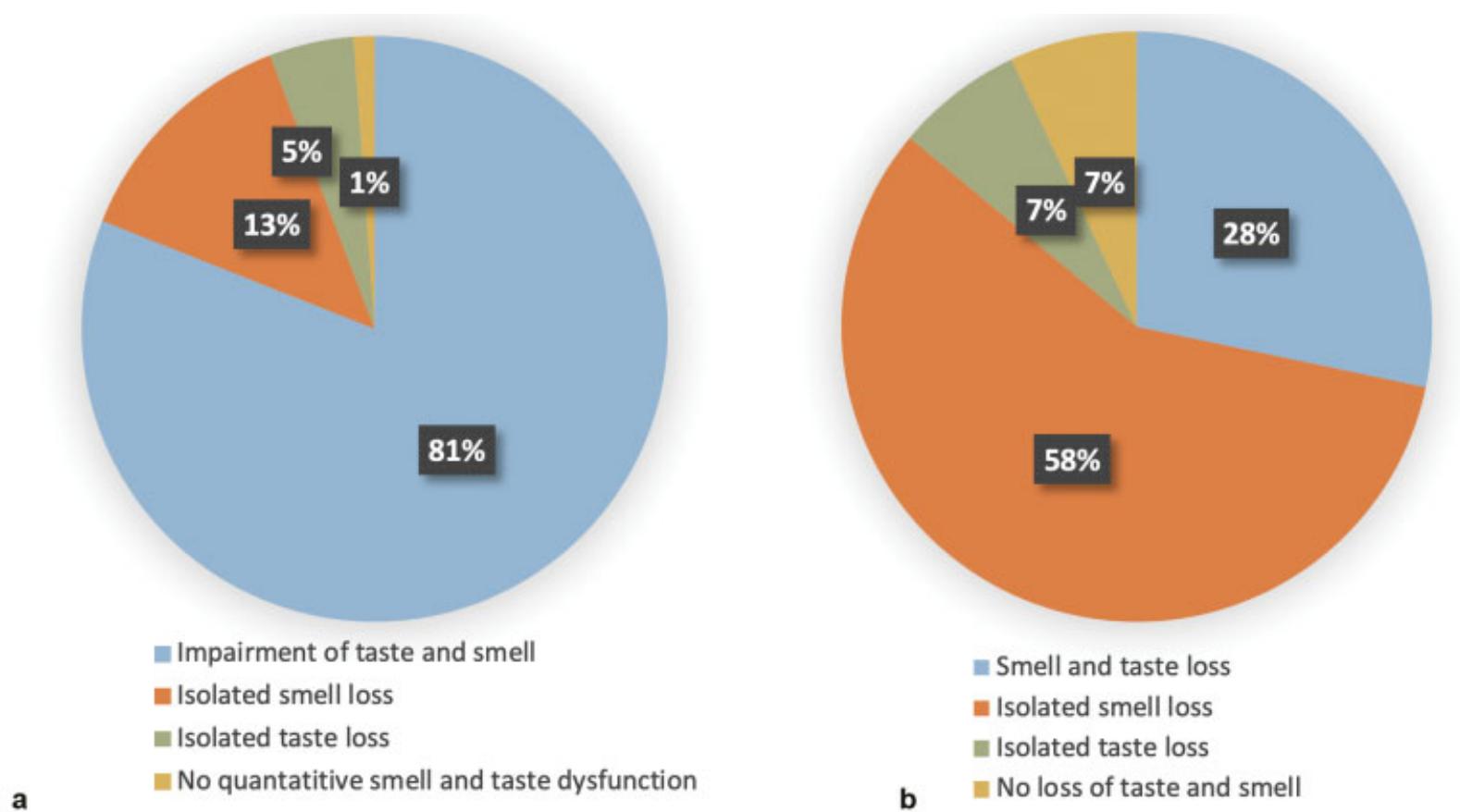

Fig. 1 (a) Distribution of the subjective olfactory and gustatory ratings $(n=258)$. The patient group underwent both smell and taste tests (Taste-Strips test or Taste-Drop-Test). Impairment of smell and taste reflects subjective self-rating as reduced or absent. (b) Distribution of the olfactory and gustatory test results $(n=258)$. The patient group underwent both smell and taste tests (Taste-Strips test or Taste-Drop-Test). Loss of smell reflects hyposmic and anosmic patients. Loss of taste reflects hypogeusic and ageusic patients.

patient group scored within the anosmic or hyposmic range, while only $12 \%$ reported olfactory dysfunction.

In the present study, the report by the patients of subjective anosmia was a strong indicator of measured anosmia or hyposmia. However, subjective olfactory complaints should be followed up with a measurement of the olfactory function for several reasons. First, it is of great importance to ensure an accurate diagnosis, since self-assessed chemosensory impairment may be overestimated or misclassified. As found in our study, $16.8 \%$ of patients with a reduced subjective sense of smell had a normal measured olfactory function, and only $64.4 \%(n=222)$ of patients with an absent subjective sense of smell were found to have lost the sense completely. Second, accurate results from a validated olfactory test are indispensable to monitor the efficacy of an applied treatment such as olfactory training. Third, there is an age-dependent loss of smell that is not reflected in the definition of hyposmia/anosmia threshold values. ${ }^{22}$ This suggests that especially elderly subjects who present themselves with loss of smell may as well perform better than expected relative to their age group. Thus, olfactory testing serves as an informative instrument to clarify whether subjective impairment reflects an actual loss of smell or a benign age-dependent condition.

\section{Accuracy of Subjective Gustatory Function in Patients}

We found that the subjective assessment of gustatory function was a good measurement of gustatory function based on test scores (Taste-Drop-Test, ROC AUC: 0.5802; Taste Strips test, ROC AUC: 0.5436 ). In total, $55 \%$ of the patients with subjective ageusia and $67 \%$ of the patients with subjective hypogeusia scored within the normogeusic range.
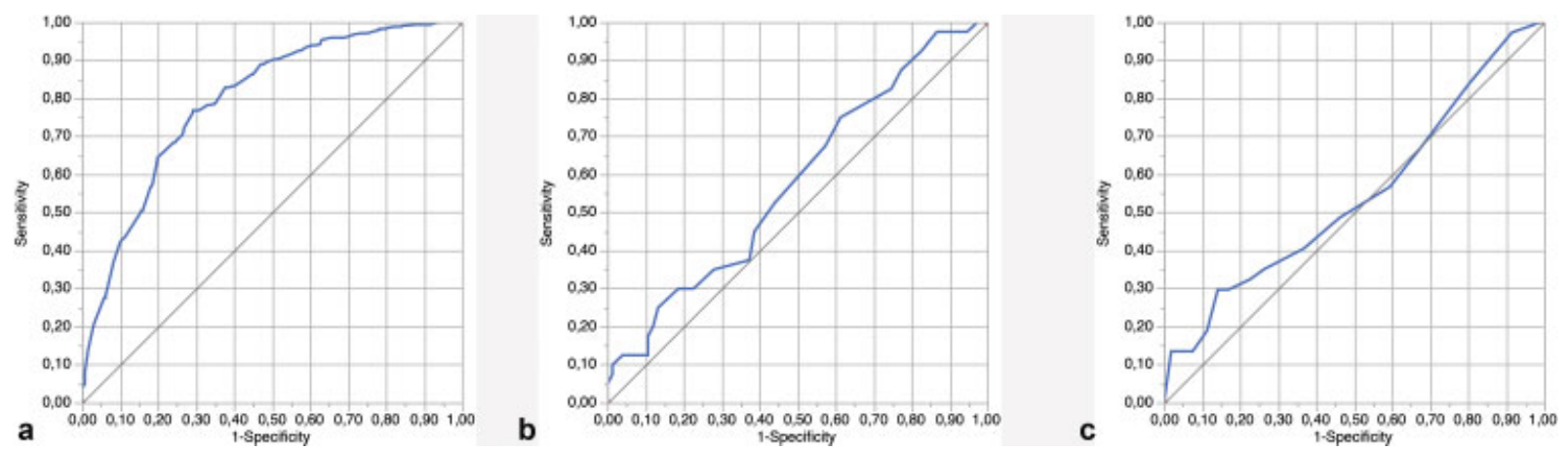

Fig. 2 Receiver operating characteristic curves that illustrate the decreased or absent subjective smell/taste function as a classifier for measured taste/smell function. (a) Subjective assessment of smell function versus the threshold, discrimination, and identification (TDI) score. (b) Subjective assessment of taste function versus the Taste-Drop-Test score. (c) Subjective assessment of taste versus Taste Strips test score. 
There are several possible explanations for these results. Gustatory impairment is relatively rare in patients with reported chemosensory dysfunction. We found that only $7 \%(n=18)$ of patients with chemosensory complaints suffered from an isolated loss of taste. This low number is consistent with previous results ranging from $1 \%$ to $4 \%{ }^{23,24}$ Another factor is that patients may have mistaken an actual olfactory deficit for a subjective loss of taste, since olfaction and gustation are interrelated, and both are vital for the perception of flavour. Also, smell dysfunction is highly frequent compared with taste disorders. ${ }^{25}$

We found that $78 \%(n=29)$ of the patients with a normal subjective taste function had a normal measured taste function. In accordance with our results, a previous study by Soter et al. ${ }^{26}$ found that the accuracy of questionnaire statements in detecting loss of taste was poor, while patients with no gustatory complaints were often correct in their statements.

However, the present study differed from the study by Soter et al. ${ }^{26}$ in several aspects. Instead of basing the diagnosis of olfactory impairment on an olfactory identification test, olfactory threshold and discrimination scores were also assessed. The questionnaire method to assess subjective chemosensory loss was different: while we asked the patients to rate the general taste and smell functions, Soter et al. ${ }^{26}$ asked the patients to rate specific scenarios of flavour perception. Furthermore, the patients in the present study were all referred from ENT specialists, while information regarding referral was not included by Soter et al. ${ }^{26}$ One could hypothesize that the risk of chemosensory misclassification is lower in patients assessed by an ENT specialist who has increased awareness of chemosensory function and related organs. With our selected cohort, these findings contribute to the findings made by Soter et al. ${ }^{26}$ In general, this suggests that patients are rather inaccurate in reporting a genuine taste problem, irrespective of being asked to rate the general taste function or regarding questions on specific tastants. Contrarily, patients reporting the non-existence of a taste problem are typically correct in their assessment, and gustatory testing is in that case unnecessary.

\section{Subjective Loss of Taste Usually Reflects Loss of Smell} We found that patients with a self-assessed impaired gustatory function ( $n=221$ ) often had a normal taste test $(n=138)$ results and a reduced measured olfactory function $(n=186 / 221,84 \%)$. This finding may suggest that the patients have a tendency to classify an olfactory impairment as a subjective taste disorder. This corresponds with the findings of a previous study, in which patients complaining of isolated taste loss were three times more prone to demonstrate an olfactory deficit than a gustatory deficit. ${ }^{24}$ Furthermore, in a recent study ${ }^{27}$ on 358 patients with subjective loss of taste, only $9.5 \%$ of patients with a combined taste and smell complaint had a measurable gustatory impairment, whereas $87 \%$ had an impaired olfactory function. However, in this study, ${ }^{27}$ the suprathreshold test for gustatory function was applied, and no olfactory discrimination test was included. This limited the interpretation of the relationship between the two senses, and highlighted the need for the present study.
The aforementioned findings may reflect that patients confuse smell impairment, taste impairment, and combined chemosensory impairment. Overall, olfactory impairment should be considered as an underlying cause when patients present with a subjective loss of taste.

\section{Limitations and Strengths of the Study}

As with prior studies, the population of patients included in the present study is a selected cohort. All patients in the present study were referred to a specialized taste and smell clinic by an ENT specialist. However, as the results are comparable with those of previous studies, the current findings highlight the argument that chemosensory testing is imperative for accurate diagnostics, irrespective of whether the patients have previously been assessed by an ENT specialist.

In the present study, cognitive function or lack of confrontation with the nature of their chemosensory deficit are not likely factors that can explain the current results. All patients were confronted with their claimed subjective chemosensory loss prior to the referral by an ENT specialist, and $99.3 \%(n=598)$ of all patients had a normal MMSE score at the time of their visit.

The study uses two different taste tests to estimate gustatory function in the patient group. Optimally, such testing should be performed with one test only to make the data more homogeneous. However, both tests are validated with good reproducibility, and are used in clinical settings.

Quantitative measures were used for the olfactory and gustatory test scores. However, such measures do not entirely cover the subjective disturbances for these senses. Distortions or phantom taste or smell sensations could also be experienced by patients, which is not always directly reflected in the olfactory test scores. ${ }^{28,29}$ This adds to the complexity of chemosensory dysfunction and calls for a combination of chemosensory-specific patient history and thorough chemosensory testing.

\section{Conclusion}

Subjective gustatory dysfunction was poorly correlated with measured gustatory dysfunction. Instead, it was often found to reflect olfactory dysfunction. Contrarily, subjective olfactory dysfunction was a more reliable measurement of olfactory dysfunction. Although subjective anosmia was a strong indicator of measured anosmia or hyposmia, the existence of remaining olfactory function was frequently found in these patients. This finding is relevant for prognosis and effects of olfactory training.

As such, validated chemosensory testing should be performed in patients with a perceived olfactory or gustatory deficit, as this could help ensure increased diagnostic precision and relevant treatment.

\section{Statement of Ethics}

The present study was conducted according to the Declaration of Helsinki on Biomedical Research Involving 
Human Subjects. As the study was based on register data, it did not require regional Ethical Committee approval in accordance with the Danish Committee Act, Section 10, Subsection 1. During enrolment, the subjects were given detailed information about all testing procedures. Written consent was obtained from all patients prior to the study, and storage of data was approved by the Danish Data Protection Agency (reference number 1-16-02-394-19).

\section{Author Contributions}

The idea for the project was conceived by the last author. Both authors contributed to the planning of the study and data analysis, wrote the initial draft of the manuscript, and contributed to a subsequent review of the manuscript.

\section{Funding Sources}

The last author wishes to acknowledge research salary funding from Arla Foods (Viby, Denmark) and the Central Denmark Region, as well as research funding from the Hans Skouby Foundation and the Velux Fonden. The sponsors had no say, roles or responsibilities in relation to the study, including (but not limited to) the study design, data collection, management and analysis.

\section{Conflict of Interests}

The authors have no conflicts of interest to declare.

\section{References}

1 Fjaeldstad A, Fernandes H, Nyengaard JR, Ovesen T. [The sense of taste in a clinical setting]. Ugeskr Laeger 2018;180(18): V08170627

2 Roper SD. Taste buds as peripheral chemosensory processors. Semin Cell Dev Biol 2013;24(01):71-79. Doi: 10.1016/j. semcdb.2012.12.002

3 Fjaeldstad A, Clausen $\mathrm{CH}$, Kjærgaard T, Ovesen T. [The forgotten cranial nerve - clinical importance of olfaction]. Ugeskr Laeger 2015;177(03):265-269

4 Vennemann MM, Hummel T, Berger K. The association between smoking and smell and taste impairment in the general population. J Neurol 2008;255(08):1121-1126. Doi: 10.1007/s00415-008-0807-9

5 Neuland C, Bitter T, Marschner H, Gudziol H, Guntinas-Lichius O. Health-related and specific olfaction-related quality of life in patients with chronic functional anosmia or severe hyposmia. Laryngoscope 2011;121(04):867-872. Doi: 10.1002/lary.21387

6 Croy I, Nordin S, Hummel T. Olfactory disorders and quality of life-an updated review. Chem Senses 2014;39(03):185-194. Doi: 10.1093/chemse/bjt072

7 Malaty J, Malaty IAC. Smell and taste disorders in primary care. Am Fam Physician 2013;88(12):852-859

8 McNeill E, Ramakrishnan Y, Carrie S. Diagnosis and management of olfactory disorders: survey of UK-based consultants and literature review. J Laryngol Otol 2007;121(08):713-720

9 Fokkens WJ, Lund VJ, Mullol J, et al. EPOS 2012: European position paper on rhinosinusitis and nasal polyps 2012. A summary for otorhinolaryngologists. Rhinology 2012;50(01):1-12. Doi: 10.4193/Rhino50E2

10 Fokkens WJ, Lund VJ, Hopkins C, et al. European Position Paper on Rhinosinusitis and Nasal Polyps 2020. Rhinology 2020;58 (S29):1-464

11 Welge-Luessen A, Hummel T, Stojan T, Wolfensberger M. What is the correlation between ratings and measures of olfactory func- tion in patients with olfactory loss? Am J Rhinol 2005;19(06): 567-571

12 Haxel BR, Bertz-Duffy S, Fruth K, Letzel S, Mann WJ, Muttray A. Comparison of subjective olfaction ratings in patients with and without olfactory disorders. J Laryngol Otol 2012;126(07): 692-697. Doi: 10.1017/S002221511200076X

13 Fjaeldstad AW, Stankovic J, Onat M, Stankevice D, Ovesen T. Patients and experiences from the first Danish flavour clinic. Dan Med J 2020;67:1-5

14 Hummel T, Sekinger B, Wolf SR, Pauli E, Kobal G. 'Sniffin' sticks': olfactory performance assessed by the combined testing of odor identification, odor discrimination and olfactory threshold. Chem Senses 1997;22(01):39-52. Doi: 10.1093/chemse/22.1.39

15 Niklassen AS, Ovesen T, Fernandes H, Fjaeldstad AW. Danish validation of sniffin' sticks olfactory test for threshold, discrimination, and identification. Laryngoscope 2018;128(08):1759-1766. Doi: 10.1002/lary.27052

16 Kobal G, Klimek L, Wolfensberger M, et al. Multicenter investigation of 1,036 subjects using a standardized method for the assessment of olfactory function combining tests of odor identification, odor discrimination, and olfactory thresholds. Eur Arch Otorhinolaryngol 2000;257(04):205-211

17 Mueller C, Kallert S, Renner B, et al. Quantitative assessment of gustatory function in a clinical context using impregnated "taste strips". Rhinology 2003;41(01):2-6

18 Fjaeldstad A, Niklassen AS, Fernandes HM. Re-Test Reliability of Gustatory Testing and Introduction of the Sensitive Taste-DropTest. Chem Senses 2018;43(05):341-346. Doi: 10.1093/chemse/ bjy019

19 Folstein MF, Folstein SE, McHugh PR. "Mini-mental state". A practical method for grading the cognitive state of patients for the clinician. J Psychiatr Res 1975;12(03):189-198. Doi: 10.1016/ 0022-3956(75)90026-6

20 Lötsch J, Hummel T. Clinical usefulness of self-rated olfactory performance - a data science-based assessment of 6000 patients. Chem Senses 2019;44(06):357-364. Doi: 10.1093/chemse/bjz029

21 Shu C-H, Hummel T, Lee P-L, Chiu C-H, Lin S-H, Yuan B-C. The proportion of self-rated olfactory dysfunction does not change across the life span. Am J Rhinol Allergy 2009;23(04):413-416. Doi: 10.2500/ajra.2009.23.3343

22 Oleszkiewicz A, Schriever VA, Croy I, Hähner A, Hummel T. Updated Sniffin' Sticks normative data based on an extended sample of 9139 subjects. Eur Arch Otorhinolaryngol 2019;276 (03):719-728. Doi: 10.1007/s00405-018-5248-1

23 Pribitkin E, Rosenthal MD, Cowart BJ. Prevalence and causes of severe taste loss in a chemosensory clinic population. Ann Otol Rhinol Laryngol 2003;112(11):971-978. Doi: 10.1177/ 000348940311201110

24 Deems DA, Doty RL, Settle RG, et al. Smell and taste disorders, a study of 750 patients from the University of Pennsylvania Smell and Taste Center. Arch Otolaryngol Head Neck Surg 1991;117 (05):519-528

25 Landis BN, Konnerth CG, Hummel T. A study on the frequency of olfactory dysfunction. Laryngoscope 2004;114(10):1764-1769. Doi: 10.1097/00005537-200410000-00017

26 Soter A, Kim J, Jackman A, Tourbier I, Kaul A, Doty RL. Accuracy of self-report in detecting taste dysfunction. Laryngoscope 2008; 118(04):611-617. Doi: 10.1097/MLG.0b013e318161e53a

27 Hunt JD, Reiter ER, Costanzo RM. Etiology of subjective taste loss. Int Forum Allergy Rhinol 2019;9(04):409-412. Doi: 10.1002/ alr.22263

28 Hong S-C, Holbrook EH, Leopold DA, Hummel T. Distorted olfactory perception: a systematic review. Acta Otolaryngol 2012;132 (1, Suppl 1):S27-S31

29 Hummel T, Whitcroft KL, Andrews P, et al. Position paper on olfactory dysfunction. Rhinol Suppl 2017;54(26):1-30. Doi: 10.4193/Rhin16.248 